\title{
Giant Cystic Chondroid Hamartoma
}

We report a case of huge pulmonary chondroid hamartoma with multilocular cysts in a 38-yr-old male patient. The lobectomy specimen of the left lower lobe showed a large multilocular cystic mass, $11.5 \times 10 \mathrm{~cm}$ in size. The mass had thin-walled, variable-sized cysts and areas of solid cartilaginous small nodules in the interstitium, which occupied the superior segment and the upper portion of the basal segment. There was no connection with bronchus or vessel. Microscopically, the cysts and cleft-like spaces were lined by ciliated columnar epithelium and the solid components were composed of cartilage, myxohyalinized connective tissue, and adipose tissue. Also seen were foci of calcification within the sclerotic stroma.

Key Words : Hamartoma; Lung Neoplasms; Cysts
Gou Young Kim, Joungho Han*, Dong Hoon Kim ${ }^{*}$, Jhingook Kim Kyung Soo Lee ${ }^{\ddagger}$

Department of Pathology, Gachon Medical School Gil Medical Center, Incheon; Department of Pathology*, Department of Thoracic Surgery ${ }^{\dagger}$ and Department of Radiology and Center for Imaging Science, Sungkyunkwan University School of Medicine Samsung Medical Center, Seoul, Korea

Received : 12 April 2004 Accepted : 20 May 2004

Address for correspondence Joungho Han, M.D.

Department of Pathology, Sungkyunkwan University School of Medicine, Samsung Medical Center, 50 Irwon-dong, Gangnam-gu, Seoul 135-710, Korea

Tel : +82.2-3410-2765, Fax : +82.2-3410-0025

E-mail : jhhan@smc.samsung.co.kr

\section{INTRODUCTION}

Hamartoma is defined as an abnormal mixture of tissue elements, or an abnormal proportion of a single element, normally present in an organ. Pulmonary chondroid hamartoma $(\mathrm{PCH})$ is the most common benign neoplasm in the lung, and accounts for $7-14 \%$ of all solitary lung nodules. However, cystic PCH is very rare (1-6). PCH of larger than $10 \mathrm{~cm}$ in size is also very rare (7).

We report a rare case of huge $\mathrm{PCH}$ with multilocular cysts in a 38-yr-old male patient.

\section{CASE REPORT}

A 38-yr-old male presented with chronic cough and dyspnea for 8 months. Dyspnea was aggravated for the previous 4 days. Chest radiography showed a large multilobulated cystic and solid mass in the left lower lobe of the lung. Chest computed tomography revealed a huge multiseptated cystic and solid mass containing foci of intralesional calcifications in the left lower lobe of the lung (Fig. 1). A left lower lobe lobectomy was done under the impression of congenital cystic adenomatoid malformation (CCAM) or lung cancer.

On gross examination, a huge cystic and solid mass containing variable size of multilocular cysts and solid component with numerous interstitial cartilaginous small nodules was found and occupied the superior segment and the upper portion of basal segment, measuring $11.5 \times 10 \mathrm{~cm}$ in size (Fig. 2A). There was no connection with bronchus or vessel. Microscopically, multilocular cystic spaces with intervening lobulated fragments of cartilaginous tissue and hyalinized stroma were seen (Fig. 2B). The cysts and cleft-like spaces were lined by ciliated columnar epithelium. There were also foci of mature adipose tissues and a few spindle cells within the intervening stroma (Fig. 2C). Also seen were foci of calcification within the sclerotic stroma. The patient recovered uneventfully and there was no evidence of recurrence for nine months after the operation.

\section{DISCUSSION}

Hamartomas are the most common benign tumors of the lung and they comprise an admixture or overgrowth of various or single normal components that should be there. Depending upon the predominant component, hamartomas can be subdivided into various subtypes; chondromatous, leiomyomatous, lymphangiomyomatous, adenofibromatous and fibroleiomyomatous. Chondromatous hamartomas are the most common subtype and have been divided into endobronchial and intraparenchymal (peripheral) lesions. The onset of the tumor is in adulthood, with the peak age incidence in the sixth decade. Hamartomas may range from 1 to larger than 
$10 \mathrm{~cm}$ in the greatest dimension, but usually are smaller than $4 \mathrm{~cm}$. One case has been reported of a tumor, measuring 16 $\times 9 \mathrm{~cm}$ in size (7). PCHs are frequently discovered on routine chest roentgenograms, in which they appear as solitary coin lesions. Less commonly, they may represent as multiple coin lesions or masses (8). However, even less frequently, cystic

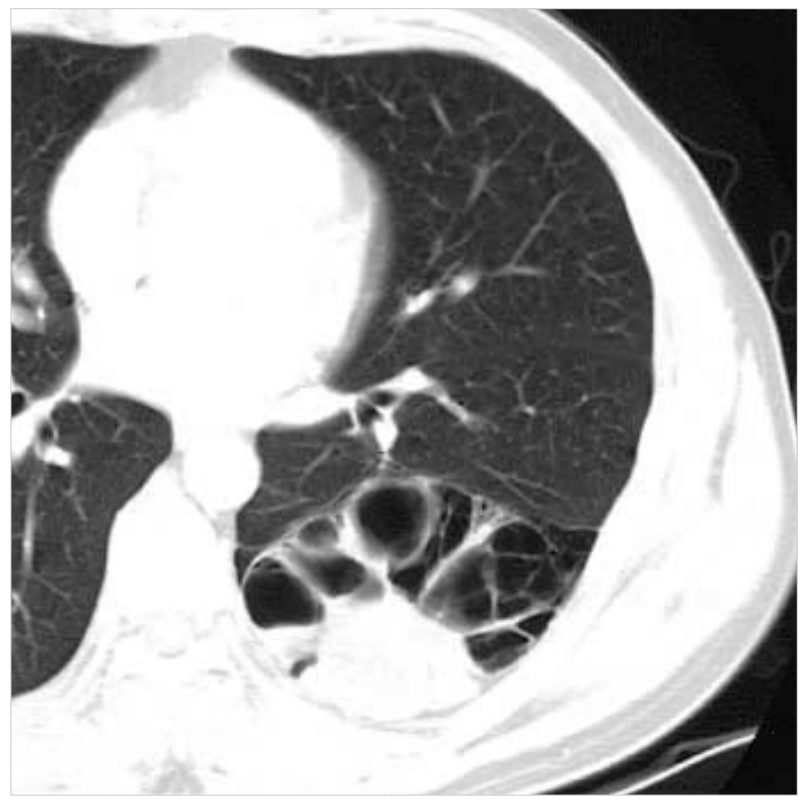

Fig. 1. Chest computed tomography reveals a huge multiseptated cystic and solid mass containing foci of intralesional calcifications in the left lower lobe of the lung.
PCH may present as cavitary lesions on chest roentgenograms (5). In these hamartomas, cystic ones are very rare (1-6).

The mechanism of cyst development within a hamartoma is unknown. The route of entry of air into these lesions could be hypothesized, and check-valve mechanism might result in the gradual expansion of small epithelial-lined tubules resembling bronchioles (3). However, our case had no bronchial connection to the air-filled multicystic area. The growth condition of PCHs resulting from that the clefts-like spaces expanding to become growing cysts was also described (5).

Rearrangement of the high mobility group (HMG) proteins, non-histone DNA binding protein, HMGIC and HMCI (Y) has been recently proposed in PCHs (9). HMGIC-LPP (lipoma preferred partner) fusion gene has been described in two histologically different tumor types; lipomas and PCHs (10).

The differential diagnosis of cystic PCH includes CCAM, mesenchymal cystic hamartoma, cystic fibrohistiocystic tumors and cystadenoma. The CCAM is thought to be due to the arrest of the bronchial tree resulting in the adenomatous appearance composed of bronchiole-like or alveolar-like spaces. The mesenchymal cystic hamartoma is composed of cystic nodules with lining of normal or metaplastic respiratory epithelium and cambium layer of primitive mesenchymal cells without normal differentiation. The fibrohistiocystic tumors are presented as primary lung tumors or metastatic tumors of cellular dermatofibromas, which show frequent cystic change. The cystic lesions are associated with interstitial spindle cell proliferation, which demonstrates a storiform pattern, produc-
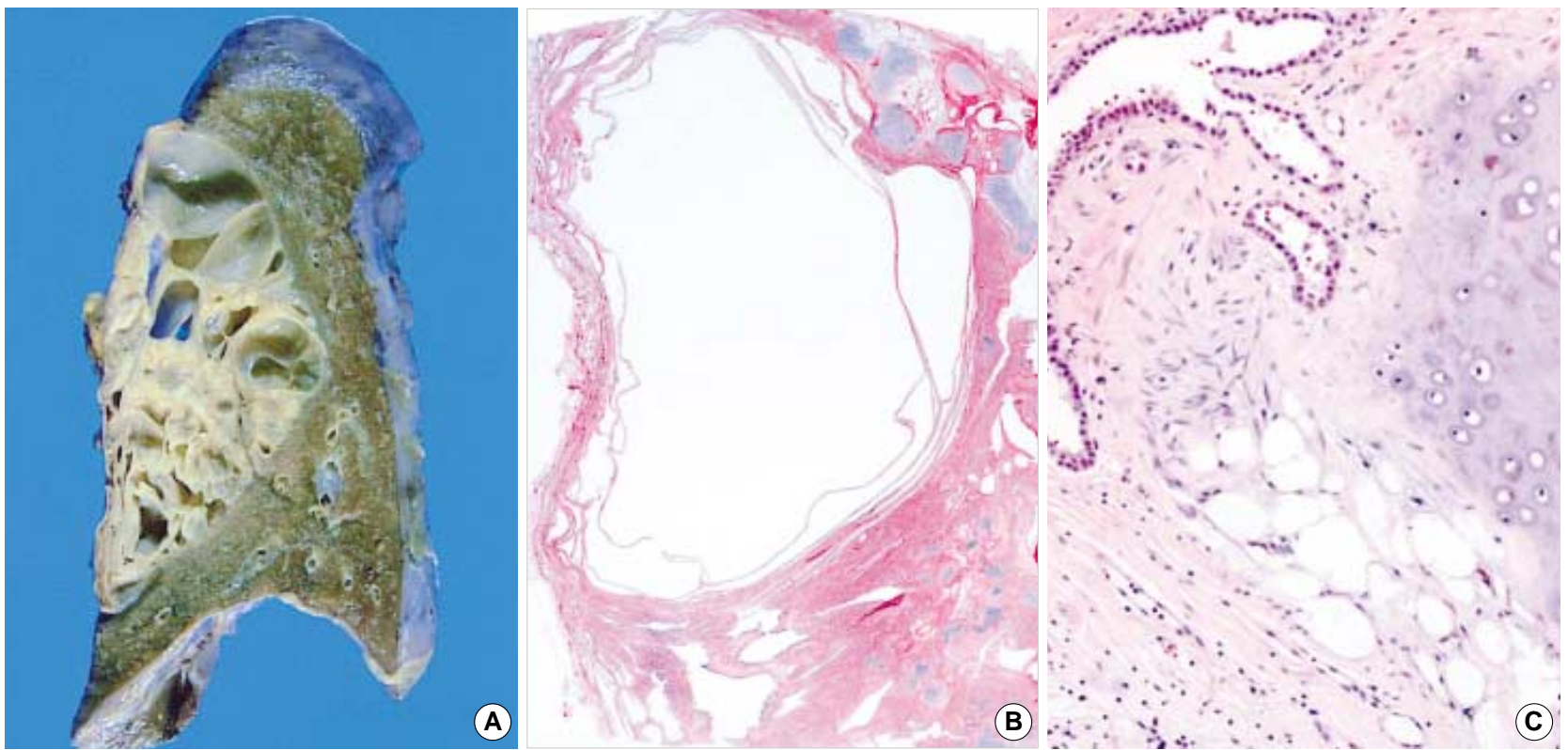

Fig. 2. (A) Gross photograph of the lower lobe of left lung shows a large cystic and solid mass containing variable size of multilocular cysts and solid component with numerous interstitial cartilaginous small nodules. (B) Multilocular cystic spaces with intervening lobulated fragments of cartilaginous tissue and hyalinized stroma (H\&E stain, original magnification $\times 1$ ). (C) There are islands of mature cartilage, adipose tissues and immature mesenchymal tissue containing spindle cells within the intervening stroma (H\&E stain, original magnification $\times 100)$. 
ing an overall impression of fibrous histiocytomas. The cystadenoma consisted of numerous irregularly arranged, tubular or dilated glands lined by a single layer of columnar, occasionally flattened, mucus-secreting cells. Hemorrhagic pattern of sclerosing hemangioma and cystic metastatic low-grade sarcomas are also included in differential diagnosis.

We may consider that the current $\mathrm{PCH}$ with huge size and multilocular cysts is exceptionally rare and should be differentiated from cystic pulmonary diseases.

\section{REFERENCES}

1. Jackson RC, McDonald JR, Clagett OT. Massive cystic pulmonary hamartoma; report of two cases. J Thorac Surg 1956; 31: 504-10.

2. Bateson EM, Abbott EK. Mixed tumors of the lung, or hamarto-chondromas. A review of the radiological appearances of cases published in the literature and a report of fifteen new cases. Clin Radiol 1960; 11: 232-47.

3. Doppman J, Wilson G. Cystic pulmonary hamartoma. Br J Radiol 1965; 38: 629-31.
4. Demos TC, Armin A, Chandrasekhar AJ, Barron J. Cystic hamartoma of the lung. J Can Assoc Radiol 1983; 34: 149-50.

5. Miura K, Hori T, Yoshizawa K, Hamaguchi N, Morita J. Cystic pulmonary hamartoma. Ann Thorac Surg 1990; 49: 828-9.

6. Austin JR, deTar M, Rice DH. Pulmonary chondroid hamartoma presenting as an inflatable neck mass. Case report and clinicopathologic analysis. Arch Otolaryngol Head Neck Surg 1994; 120: 440-3.

7. Lee SY, Park HJ, Lee CS, Lee KR. Giant pulmonary hamartoma. Eur J Cardiothorac Surg 2002; 22: 1006.

8. King TE Jr, Christopher KL, Schwarz MI. Multiple pulmonary chondromatous hamartomas. Hum Pathol 1982; 13: 496-7.

9. Kazmierczak B, Rosigkeit J, Wanschura S, Meyer-Bolte K, Van de Ven WJ, Kayser K, Krieghoff B, Kastendiek H, Bartnitzke S, Bullerdiek J. HMGI-C rearrangements as the molecular basis for the majority of pulmonary chondroid hamartomas: a survey of 30 tumors. Oncogene 1996; 12: 515-21.

10. Rogalla P, Lemke I, Kazmierczak B, Bullerdiek J. An identical HMGIC-LPP fusion transcript is consistently expressed in pulmonary chondroid hamartomas with t(3;12)(q27-28;q14-15). Genes Chromosomes Cancer 2000; 29: 363-6. 